\title{
New Particle Searches at LEP
}

\author{
Adriaan Buijs
}

CFRN, Geneva

\section{Introduction}

Since the startup of the LEP accelerator, providing $e^{+} e^{-}$collisions at centrcof-mass energies corresponding to the mass of the $Z^{0}$, many searches for new particles have been performed by the four big experiments $\Lambda$ LEPII, L3, DELPHI and OPAL.

In this talk 1 will describe a few of these searches: the search for the sixth quark, and for a new quark from a possible fourth family; the search for the Standard Model Higgs and for Higgs doublets from Super Symmetric models; and the search for excited quarks and leptons. The techniques used in these searches are representative for other searches not described in this talk. The MARK II collaboration at the SLC has performed a series of searches as well. In spite of the low statistics, some of the limits obtained at the SLC are comparable to those from LEP and close to the kinematic limit. However, they are not part of this talk.

In anticipation of the conclusion section I should say that no new particles have been found at LEP, and therefore all results are expressed in terms of lower limits, mostly at the $95 \%$ confidence level.

The dala samples used by the four LEP experiments vary in size, but correspond largely to the running periods in 1989 and the first part of 1990 up to this conference. Since then the limits have been raised, in particular 
for the Higgs searches, but here the status of the analyses at the time of the conference will be given.

The talk will be divided into indirect searches using the $Z^{0}$ resonance parameters and direct searches using particular event topologies to identify new particles.

\section{Indirect Searches}

The resonance parameters of the $Z^{0}$ have been measured by all four experiments to great accuracy during the first running periods of LEP. They were recently summarised by $E$. Fernandez.[1]

The total $Z^{0}$ width is the sum of the partial widths $\Gamma_{2}=\Gamma_{\text {had }}+\Gamma_{1+1-}+$ $\Gamma_{\text {inx }}$. Here $\Gamma_{\text {hed }}$ is measured through the peak cross section of multi hadronic $Z^{0}$ decays $\sigma_{\text {had }}^{\circ}=2 \pi \Gamma_{\text {had }} \Gamma_{\text {ee }} / m_{z}{ }^{2} \Gamma_{z}{ }^{2}$, and $\Gamma_{1+1-}$, the leptonic width is measured through the lineshape of the $Z^{0}$ decays to electron, muon and tau pairs. The invisible width $\Gamma_{\text {inv }}$ contains the contributions from the neutrino families plus any additional invisible decays of the $Z^{0}$. In the Standard Model each neutrino family will contribute $165 \mathrm{MeV}$ to $\Gamma_{\mathrm{inv}}$. From a combination of all four LEP experiments with a total of approximately $185000 Z^{0}$ decays, the number of light neutrino families is found to be $n_{\nu}=2.95 \pm 0.11$. Along the lines of an early ALEPH publication [2], I will assume that there are three light neutrino families and take the error in $n_{\nu}$ as possible contribution from new invisible decays. A new decay must contribute less than

$$
\Gamma_{\mathrm{x}}<0.11 \times 1.64 \times \Gamma_{\nu_{\mathrm{e}}}=30 \mathrm{MeV},
$$

where the factor 1.64 is due to the one-sided $95 \%$ confidence level limit. For a fourth generation heavy stable neutrino $\nu_{L}$ the partial width is given by

$$
\Gamma_{\eta 2}=\frac{G_{F} m_{\mathrm{Z}}{ }^{3}}{16 \pi \sqrt{2}}\left(3 \beta+\beta^{3}\right)
$$

where $G_{F}$ is the Fermi constant. The velocity $\beta$ of the neutrino is a function of its mass: $\beta^{2}=1-4 m_{\eta}^{2} / m_{\mathrm{Z}}{ }^{2}$ and (1) and (2) therefore lead to a lower limit: $m_{\mu_{2}}>44.3 \mathrm{GcV}$.
The uncertainty in the width measurement can also be used to obtain a limit on the mass of a visible decay, for example $Z^{0} \rightarrow t \bar{t}$, where the $t$ and $\bar{t}$ fragment into hadron jets. The measurement of the peak cross section $\sigma_{\text {hed, }}$ which is proportional to $\Gamma_{\text {had }} / \Gamma_{z}^{2}$ would then be

$$
\sigma_{\text {had }} \propto \frac{\Gamma_{s q}+c \Gamma_{t}}{\left(\Gamma_{S M}+\Gamma_{t}\right)^{2}}
$$

where $\Gamma_{b q}$ is the expected hadronic width for five quarks, $\Gamma_{S M}$ is the expected (Standard Model) total width of the $\mathrm{Z}^{0}$ for five quarks and $\Gamma_{t}$ the unknown contribution from the top quark. The efficiency $\epsilon$ to observe $t \bar{t}$ events influences $\sigma_{\text {had }}$, but can be assumed to be one. Expression (3) must now be less than $\Gamma_{b q} /\left(\Gamma_{S M}+\Gamma_{X}\right)^{2}$, where $\Gamma_{x}$ is given in (1). Using the Standard Model expression for $\Gamma_{1}\left(m_{t}\right)$ and solving for $m_{t}$ we find a lower limit for the top quark mass of $m_{1}>34.9 \mathrm{GeV}$.

The L3 collaboration has given a more definite measurement of the top quark mass using the relationship between $\sin ^{2} \theta_{W}=1-m_{W}^{2} / m_{Z}{ }^{2}$ and $m_{t} \cdot[1]$ The top quark influences the value of $\sin ^{2} \theta_{w}$ through electroweak radiative corrections to the propagators of the $Z^{0}$ and $W^{ \pm}$.[3] With the $Z^{0}$ mass measured by $L 3, m_{\mathrm{Z}}=91.148 \pm 0.017 \mathrm{GeV}$, and a combined measurement of $\sin ^{2} \theta_{w}$ by the neutrino and collider experiments, $\sin ^{2} \theta_{w}=0.228 \pm 0.0045$, the top mass falls in the range $m_{\mathrm{t}}=141_{-42}^{+35} \mathrm{GeV}$. However, these limits are only to $68 \%$ confidence level. At $95 \%$ confidence level, the upper limit is raised to $200 \mathrm{GeV}$.

\section{Direct Searches}

If the topolugies of events with new particles are sufficiently distinct from the regular $Z^{0}$ decays, direct searches can give better limits than those obtained from the width measurement. The experiments in general search for new particles using topologies which give the optimal signal to background ratio for their detector. In the following sections direct searches for top, $b^{\prime}$, Higgs and excited quarks and leptons will be discussed. The discussion does not cover all search techniques used by all experiments. The cuts quoted in selection criteria are those essential to the final result; in addition many 
other preselection cuts were applied to the data, for which the reader is referred to the original publication.

\subsection{Search for Top, $b^{\prime}$}

Direct searches were performed by OPAL [4], ALEPH [2] and DELPHI [5] for the sixth quark and for a possible bottom quark of a fourth family. The regular weak decays $t \rightarrow b W^{*}$ and $b^{\prime} \rightarrow c W^{*}$ were considered in all searches, but also the flavour changing neutral current decays of the $b^{\prime}, b^{\prime} \rightarrow b \gamma$ and $b^{\prime} \rightarrow b-g l u o n$ and the decays via a charged Higgs, $t \rightarrow b H^{+}$and $b^{\prime} \rightarrow \mathrm{cH}^{-}$ were considered.

The OPAL collaboration has used an event shape analysis to search for $t$ and $b^{\prime}$. The decay of the $Z^{0}$ into heavy quarks leads to a more isotropic distribution of the final state particles, because the boost from the quark motion is less than in the decay to light quarks. OPAL has chosen the acoplanarity $A=4 \min \left(\sum_{i}\left|p_{i \perp}\right| /\left|p_{i}\right|\right)^{2}$ as a measure to separate $t$ and $b^{\prime}$ decays from the light quark decays. Here $p_{i \perp}$ is the transverse momentum of a particle with respect to the plane which minimises $A$. Figure la shows the acoplanarity distribution for 15000 multihadronic events as data points. The shaded histogram represents the expectation from the JETSET Monte Carlo with five quark flavours, whereas the open histogram gives the signal expected in the presence of a top quark of $35 \mathrm{GeV}$ mass. Such a signal is clearly incompatible with the data.

The ALEPH collaboration has looked for events with isolated charged tracks with momentum transverse to the thrust axis of at least $3 \mathrm{GeV}$. These isolated charged tracks are experted from the leptonic decay of the $W^{* *}$. An isolation parameter

$$
\rho_{i}=\min _{i} \sqrt{2 E_{i}\left(1-\cos \theta_{i j}\right)}
$$

was defined, where $E_{i}$ is the energy of particle $i$ and $\theta_{i j}$ the angle between the axis of the jet $j$ and that particle. The minimum is taken over all jets $j$ in the event. Figure $1 \mathrm{~b}$ shows the distribution of the isolation parameter for 11550 events together with the five flavour Monte Carlo (shaded histogram)
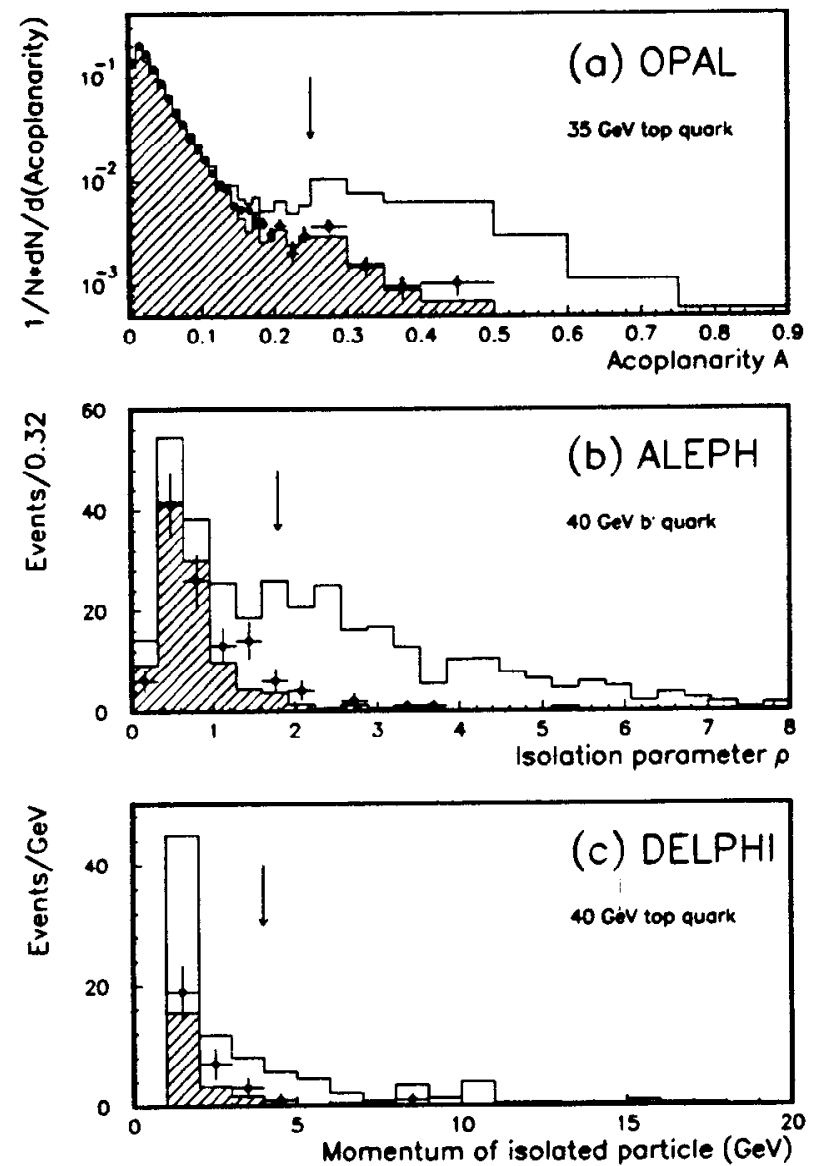

Figure 1: Distributions of parameters used in the searches for the top and $b^{\prime}$ quarks. (a) The acoplanarity spectrum by OPAL. (b) The isolation parameter distribution by ALEPH. (c) The momentum of an isolated particle by DELPHI. In all plots, the data are given as points with error bars, the shaded histogram represents a standard five-quark histogram and the open histogram a five-quark Monte Carlo with an additional quark. The arrows indicate where the collaborations plare the cuts. 
and the Monte Carlo for five flavours plus a $b^{\prime}$ quark with $40 \mathrm{GeV}$ mass (open histogram). Also this signal is incompatible with the observed events.

The DELPHI collaboration emphasised the possible $t$ and $b^{\prime}$ decays to charged Higgs bosons. The subsequent decay of the Higgs to $\tau \nu_{r}$ would give again the distinct signature of a high momentum isolated charged track. The charged track had to be separated by more than $35^{\circ}$ from the other particles in the event. Figure 1c shows the momenta of isolated particles for data, for a five quark Monte Carlo (shaded histogram) and for a Monte Carlo containing in addition a $40 \mathrm{GeV}$ top quark (open histogram). There is no signal visible in the data.

The final results obtained by the three experiments are summarised in table 1 . The production of $t$-quark and $b^{\prime}$-quark pairs is essentially excluded up to the kinematical limit of $m_{\mathrm{z}} / 2$.

\begin{tabular}{|l|ccc|}
\hline Channel & OPAL & ALEPH & DELPHI \\
\hline$t \rightarrow b W^{*}$ & 45.1 & 45.8 & 44.5 \\
$b^{\prime} \rightarrow c W^{*}$ & 45.4 & 46.2 & 45.0 \\
$b^{\prime} \rightarrow b g$ & 45.4 & 46.0 & \\
$b^{\prime} \rightarrow b \gamma$ & 46.2 & 46.0 & \\
$t \rightarrow b H^{+}$ & 45.3 & 45.3 & 44.0 \\
$b^{\prime} \rightarrow c H^{-}$ & 45.5 & 45.4 & 44.5 \\
\hline
\end{tabular}

Table 1: Summary of lower limits on the masses of the top and $b^{\prime}$ quarks from event topology searches at the $Z^{0}$. The limits are in GeV at the $95 \%$ confidence level.

\subsection{Search for the Standard Model Higgs}

In the Standard Model (SM), a neutral boson with undetermined mass is necessary to give mass to the Gauge bosons. The search for this particle, the Higgs boson, is an essential test of the Standard Model and therefore one of the main goals of the LEP experiments. (See for example, ref. [6].) In all searches the Higgs production mechanism is taken to be the Bjorken process, in which the $\mathrm{Z}^{0}$ decays into a Higgs and a virtual $\mathrm{Z}^{0}: \mathrm{Z}^{0} \rightarrow \mathrm{HZ}^{\mathrm{O*}}$ The coupling of the Higgs to bosons is proportional to their masses, and this mechanism is therefore by far dominant. In the Born approximation the differential cross section for Higgs production is (see for example ref. [7])

$$
\frac{d \sigma_{0}}{d E_{H}}=\frac{4 \alpha^{3}}{g} g^{2}\left(c_{v}^{2}+c_{a}^{2}\right)^{2} m_{Z}^{2} p_{H} \frac{12 E_{b}^{2}-12 E_{b} E_{H}+E_{H}^{2}+2 m_{H}^{2}}{R(s) R\left(s_{1}\right)} .
$$

Here $E_{H}$ and $p_{H}$ are the energy and momentum of the Higgs, $E_{b}$ the beam energy, $g=\left(\sin \theta_{W} \cos \theta_{W}\right)^{-1}, c_{v}$ and $c_{a}$ are the electroweak coupling constants. The energies $s$ and $s_{1}$ correspond to the mass squareds of the $Z^{0}$ and $\mathrm{Z}^{0 *}$, respectively. The fact of having the two resonances leads to the two terms $\mathrm{R}$ in the denominator of $(5): R(s)=\left(s-m_{\mathrm{Z}}\right)^{2}+m_{\mathrm{Z}}{ }^{2} \Gamma_{\mathrm{z}}{ }^{2}$. As a. result the cross section is highest for a light Higgs and drops off rapidly However, when one goes to higher beam energies the $\mathrm{Z}^{0 *}$ becomes real and the cross section for a heavy Higgs rises again. This is a reason to search for a heavy Higgs at LEP 200. The total cross section for Higgs production via the Bjorken mechanism is given in figure 2. The production rate for $\mathrm{Z}^{0} \rightarrow \mathrm{HZ}^{\mathrm{v}^{*}} \rightarrow \mathrm{H}^{+} \mu^{-}$is shown relative to $\mathrm{Z}^{0} \rightarrow \mu^{+} \mu^{-}$. The relative magnitudes of the Higgs decays to $b \bar{b}, c \bar{c}$ and $\tau^{+} \tau^{-}$are indicated by the dashed, the dot-dashed and the dotted lines respectively. The rate is almost $1 \%$ of the dimuon rate at low masses.

The decay of the Higgs proceeds dominantly through heavy fermions:

$$
\Gamma(H \rightarrow f \bar{f})=N_{c} \frac{g^{2} m_{f}^{2} m_{H}}{32 \pi m_{W}^{2}} \beta_{f}^{3},
$$

where $N_{c}$ is the number of colours, $m_{f}$ the fermion's mass and $\beta_{f}$ its velocity in the Higgs frame. Thus the Higgs decays mainly to the heaviest particle for which its mass is above threshold. A near-zero mass Higgs will decay exclusively to photon pairs through quark and gauge boson loops. The Higgs life time is inversely proportional to its decay width, which means that very light Higgs particles will decay well outside the LEP detectors. The virtual $Z^{0 *}$ decays as the normal $Z^{0}$ in quarks, neutrinos or leptons. 


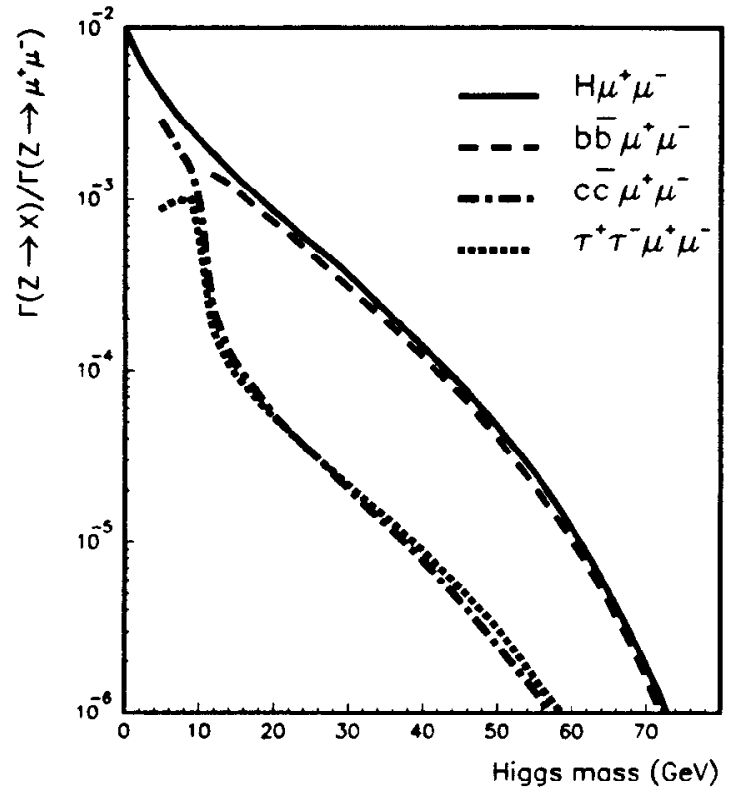

Figure 2: The rate of the process $\mathrm{Z}^{0} \rightarrow \mathrm{H} \mu^{+} \mu^{-}$via the Bjorken mechanism relative to the process $Z^{0} \rightarrow \mu^{+} \mu^{-}$, as a function of the Higgs mass. The solid line gives all $H$ decays, the dashed, dot-dashed, and dotted lines gives the contributions from Higgs decay to $b \bar{b}, c \bar{c}$ and $\tau^{+} \tau^{-}$, respectively.
The search for the Higgs boson relies heavily on Monte Carlo calculations to simulate the event topologies of Higgs decays. All experiments used the improved Born approximation [8], added initial state radiation and QCD effects and considered the dependence on the LEP beam energy. Fortunately, the influence of the top quark could be ignored.

The Higgs mass range covered by the LEP experiments was naturally split in three: $i$ ) the range from zero to $2 m_{\mu}$. In this mass range, the Higgs is long lived and decays to photon pairs or electrons; ii) the range between $2 m_{u}$ and 2 to $3 \mathrm{GeV}$, or $2 m_{c}$. The search in this range is difficult due to the theoretical uncertainty in the decay; iii) the mass range above $2 m_{c}$, where the Higgs decays into two heavy-quark jets or tau pairs.

ALEPH [9] and OPAL [10] have analysed the Higgs mass range between ero and $2 m_{\mu}=212 \mathrm{MeV}$. Both collaborations have searched for events of the type $\mathrm{Z}^{0} \rightarrow \mathrm{HZ}^{0 *}$ where $\mathrm{Z}^{0 *} \rightarrow l^{+} l^{-}$and the Higgs decay $\mathrm{H} \rightarrow \gamma \gamma$ or $e^{+} e^{-}$ occurs outside the detector. The signature for this reaction is an acoplana lepton pair without additional energy. ALEPH required an acoplanarity greater than $30 \mathrm{mrad}$ and less than $1 \mathrm{GeV}$ of isolated electromagnelic or hadronic energy. OPAL required $\sin (\theta) \phi_{\text {acop }}$ to be larger than $35 \mathrm{mrad}$ where $\theta$ is the average polar angle of the leptons and $\phi_{\text {acop }}$ the acoplanarity angle in the $x-y$ plane. To reject very asymmetric tau-pair decays, both ALEPH and OPAL applied cuts on the momenta of the leptons as well. The graphs labeled (a) in figure 3 show the number of events expected from a Higgs decay when these cuts are used. These curves were obtained from Monte Carlo calculations and were reduced by the magnitude of the systematic error to obtain conservative limits

As the Higgs mass increases more decays occur inside the detector, reducing the sensitivity. A I.EPH searched for events with a displaced vertex in the central detector (TPC). Events were used in which the $Z^{0 *}$ decayed either to neutrinos or quark pairs. The backgrounds to this search are photon conversions, and in the case of the $Z^{0 *} \rightarrow q \bar{q}$ events, also $k^{0}$ and $\Lambda$ decays. These backgrounds were removed. One event was found leading to an upper limit on the signal of 4.7 events. OPAL has searched only in events in which the $Z^{0 *}$ decays to $\nu \bar{\nu}$. One event was found, which is compatible with $Z^{0} \rightarrow \nu \bar{\nu} \gamma$. The number of events expected from the visible 

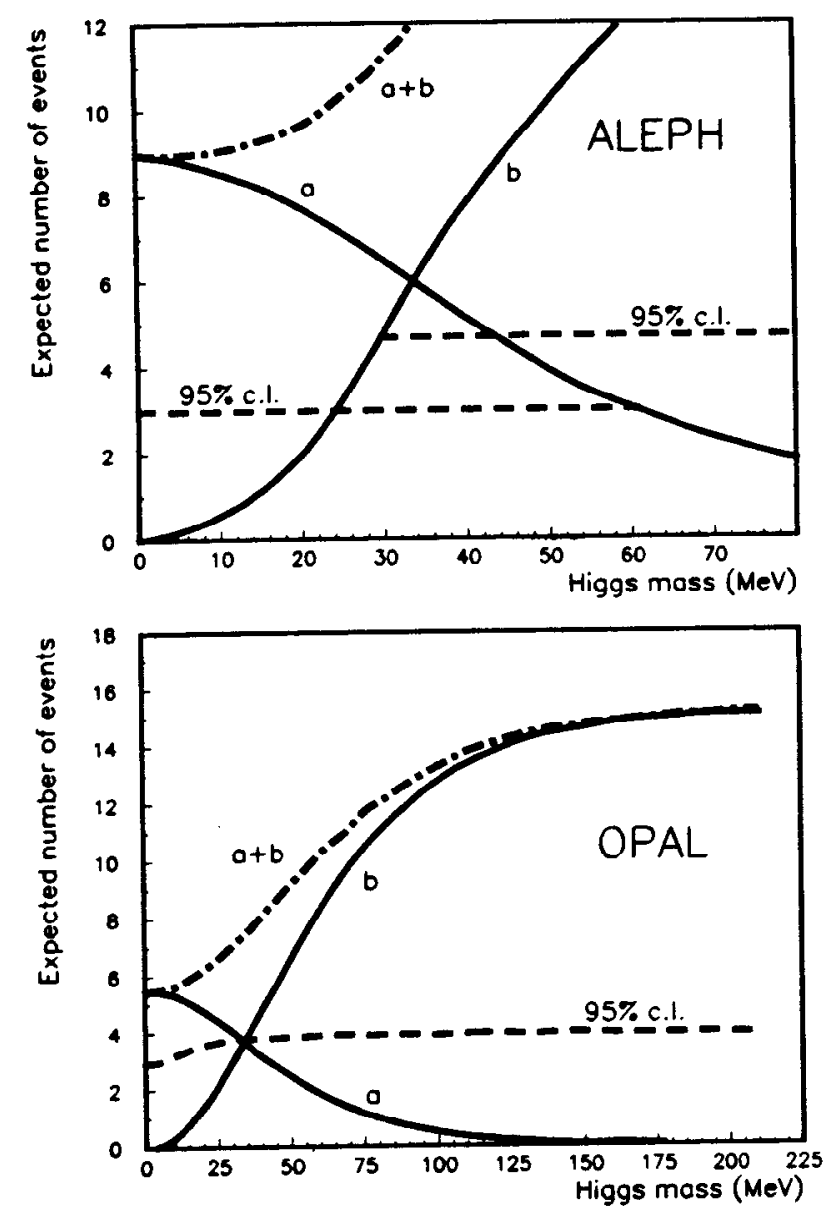

Figure 3: The numbers of low-mass Standard Model Higgs events expected by the ALEPH collaboration (top) and the OPAL collaboration (bottom). The curves labeled a represent the invisible Higgs decays (outside the detector), the curves $b$ represent the long-lived but visible decays of the Higgs (inside the detector). The curves $a+b$ give the sum of the two channels. The dashed curves give the $95 \%$ c.l. upper limit level on the number of observed events.
H decays is shown as graphs (b) in figure 3 . The dot-dashed graphs labeled $(a+b)$ give the sum of both channels. The dashed lines indicate the $95 \%$ confidence level upper limits on a observed signal, for both experiments well below the expected signals. The mass range between zero and $2 m_{\mu}$ is therefore excluded by both experiments.

The mass range between $2 m_{\mu}$ and $2 m_{c}$ was covered by DELPHI [11] and ALEPH.[12] In this mass range there is a theoretical uncertainty regarding the decay of the Higgs.[13] Both ALEPH and DELPHI have made the reasonable assumption that the Higgs will decay to a few hadrons. These few particles will appear as a low multiplicity jet as a result of the boost of the Higgs. DELPHI has used the LUND jet finding algorithm to select three-jet events. The two main jets were required to be more than $120^{\circ}$ apart and the third jet was required to have two to four tracks and satisfy kinematical constraints to be compatible with a Higgs. DELPHI found no candidate in this mass range out of 13000 events ALEPH has searched for the Higgs decaying to two particles in conjunction with lepton pairs, quark jets and neutrino pairs. They found no candidates in 11550 events. Therefore, the mass range between $212 \mathrm{MeV}$ and $3 \mathrm{GeV}$ is excluded by both DELPHI and ALEPH.

A major background to Higgs production between $212 \mathrm{MeV}$ and $3 \mathrm{GeV}$ is radiation from the final state leptons or quarks. The cross section for $q \bar{q}$ production from a virtual photon radiated by a lepton has been calculated.[14] The total width for the process $\mathrm{Z}^{0} \rightarrow l^{+} l^{-} q \bar{q}$ is of the order of $2.3 \mathrm{MeV}$. The spectrum is strongly peaked at zero invariant mass for the $q \bar{q}$ pair. However, these calculations do not take into account the hadronic nalure of the photon. An enhancement of $\rho^{0}$ production has been observed in photon-photon scattering in the reaction $\gamma \gamma \rightarrow \rho^{0} \rho^{0} \rightarrow \pi^{+} \pi^{-} \pi^{+} \pi^{-}$(see for example ref. [15]). This enhancement is still not well understood, but believed to be a feature of the Vector Dominance Model (VDM) in which the photon manifests itself as neutral vector mesons (V). One prediction of VDM is the ratio of $\rho^{0}$ mesons produced relative to $\omega$ and $\phi$ mesons, which is 1: 0.09: 0.18. The OPAL collaboration has shown a few candidate events of the type $e^{+} e^{-} \rightarrow l^{+} l^{-} \gamma^{*} \rightarrow l^{+} l^{-} V$. One such event is shown in figure 4a. Clearly visible are the two highly energetic muons, the tracks labeled 1 and 


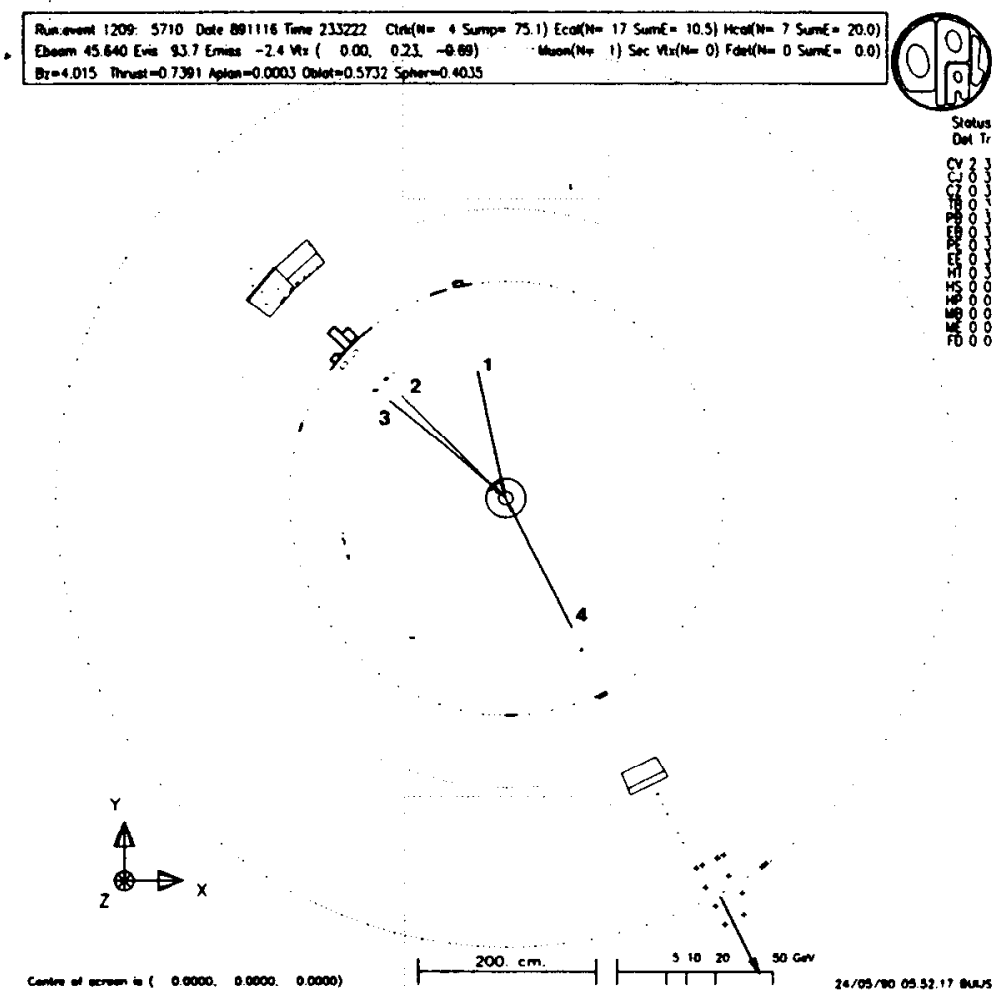

Figure 4: OPAL event, candidate for the reaction $Z^{0} \rightarrow \mu^{+} \mu^{-} \gamma^{*} \rightarrow \mu^{+} \mu^{-} \rho^{0}$. The particles labeled 2 and 3 are compatible with pions or kaons, particles labeled 1 and 4 are muons.
4. The particles labeled 2 and 3 are pions or kaons because they deposit energy in the hadron calorimeter as well as in the electromagnetic calorimeter. Furthermore, the energy loss of the particles in the central detector is compatible with that expected from pions. The invariant mass of the $\pi^{+} \pi^{-}$ pair is compatible with a $\rho^{0}$ meson.

For the Higgs mass range above $3 \mathrm{GeV}$ all experiments initially used the same purely topological search: the Higgs was assumed to decay to $b \bar{b}$ or $c \bar{c}$ jets, and the the virtual $Z^{0}$ either to neutrino pairs or to lepton pairs. The signature for the first case, the "missing energy" topology, are two jets with large momentum unbalance. For the second case, the "dilepton" topology, the events have again two jets, but the energy is balanced by two leptons. The background for the dilepton search is again from final state virtual photons decaying to a lepton pair, but now a cut on the invariant mass of the recoil system can be made to remove them. Figure 5 shows such an event observed by the DELPHI collaboration. Clearly visible are two jets, and two isolated muons emerging to the left.

OPAL [16] has applied the following cuts for its missing energy search: at least five charged tracks with a total transverse momentum unbalance of more than $6 \mathrm{GeV}$; total visible energy less than $60 \%$ of the centre-of-mass energy; the two final cuts, illustrated in figure 6 , are that more than $70 \%$ of the visible energy $E_{v i}$, be contained in a $150^{\circ}$ cone (the forward cone), whereas a backward cone of $120^{\circ}$ could contain only $2.5 \mathrm{GeV}$. Figure 6a shows $E_{\text {forward }} / E_{\text {vi }}$ for a Monte Carlo simulation of a $24 \mathrm{GeV}$ Higgs, figure $6 \mathrm{~b}$ shows the same distribution for the data. The boxes indicate the selected regions and it is clear that a $24 \mathrm{GeV}$ Higgs can be excluded.

The L3 [17] cuts for the $Z^{0} \rightarrow H_{\mu^{+}} \mu^{-}$search are: at least one muon with $p_{\mu}>15 \mathrm{GeV}$ and a second with $p_{\mu}>5 \mathrm{GeV}$; no particles in a cone of $35^{\circ}$ around at least one muon; at least two particles (of which one charged) outside a cone of $8^{\circ}$ around each muon.

Using these and similar cuts, all four LEP experiments calculated the number of events they expected as a function of the Higgs mass. The number of expected events is given in figure 7 for OPAL [16], ALEPH [12], L3 [17] and DELPHI.[11] No events were observed by either collaboration, 


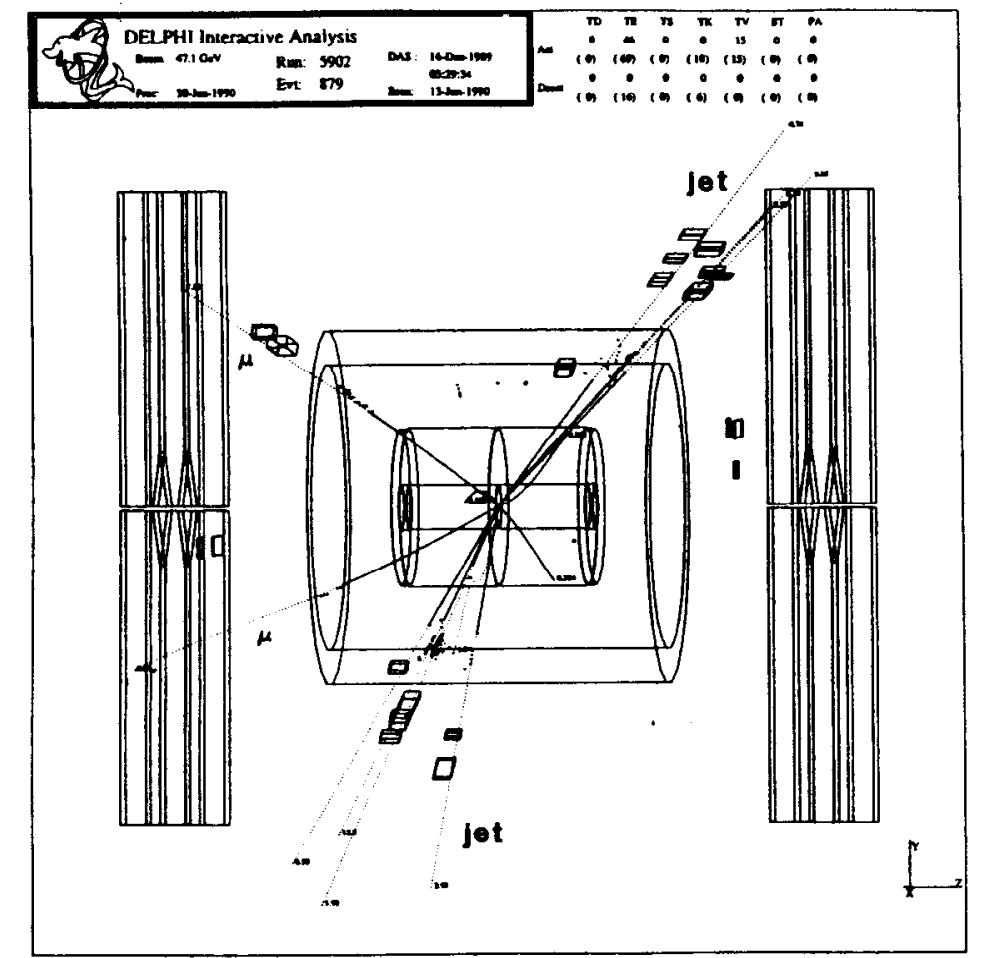

Figure 5: A candidate event from the DELPHI detector for the reaction $Z^{0} \rightarrow q \bar{q} \gamma^{*} \rightarrow j e t-j e t-\mu^{+} \mu^{-}$. The muons emerge to the left, whereas two hadron jets are discernable at the right and bottom.
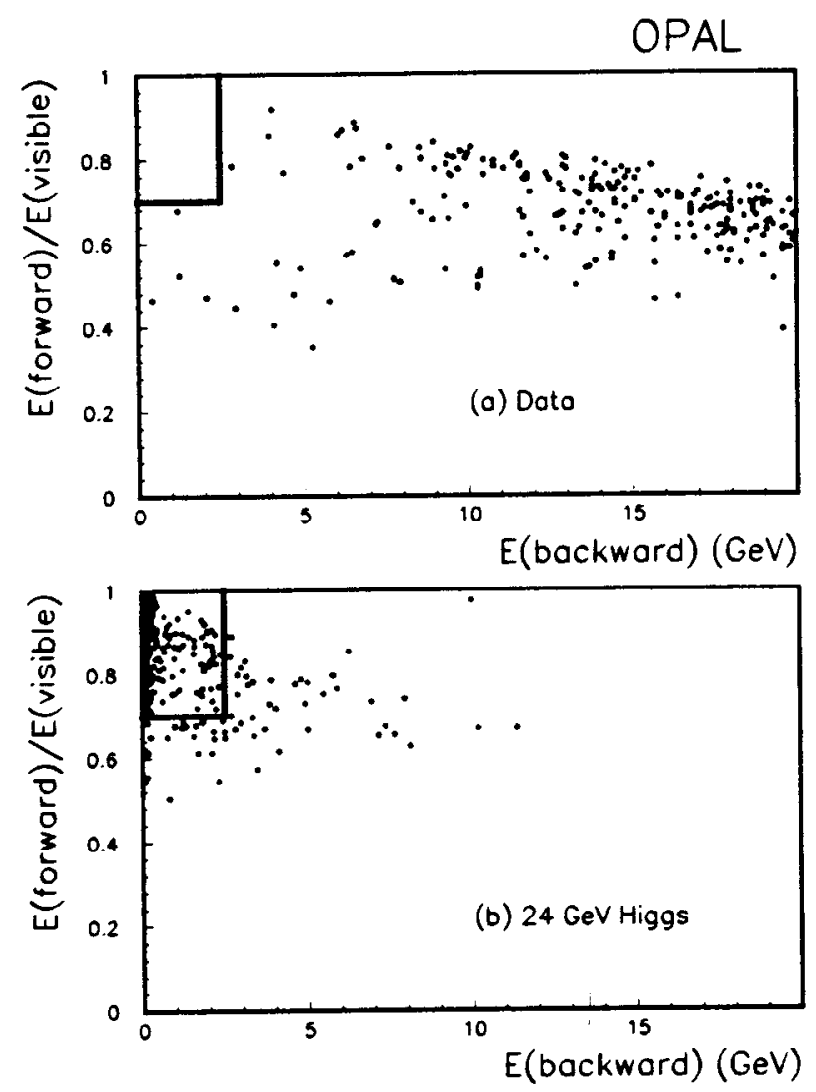

Figure 6: Final distributions in the OPAL Standard Model Higgs search The ratio of the total energy in the forward cone to the total energy in the event is plotted vs the energy in the backward cone for (a) multihadronic events, and (b) a simulation of a $24 \mathrm{GeV}$ Higgs boson. The rectangle in the upper left corner indicates the selected region. 


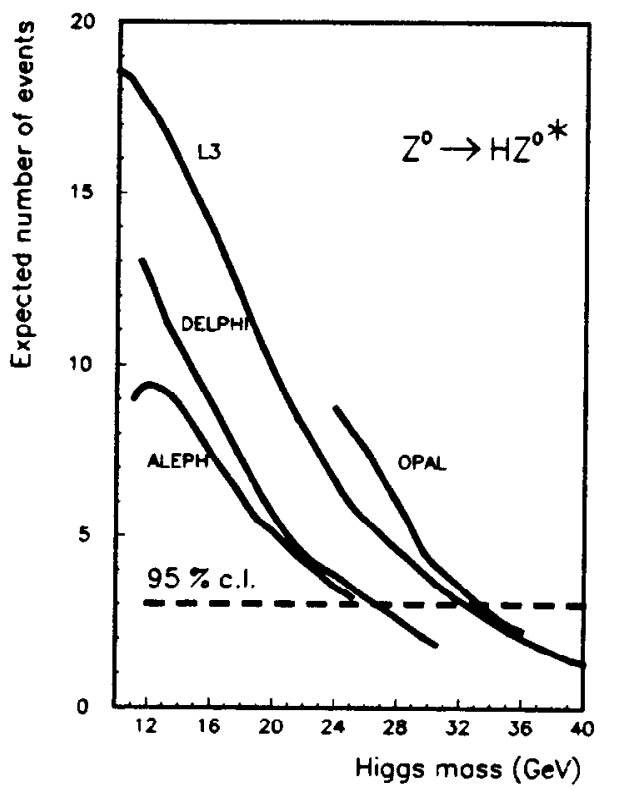

Figure 7: Expected number of events from a topological search in the combined channels $\mathrm{HZ}^{0 *} \rightarrow \mathrm{H} \nu \bar{\nu}$ and $\mathrm{HZ}^{0 *} \rightarrow \mathrm{Hl}^{+} \mathrm{l}^{-}$by the four LEP experiments as a function of the Higgs mass. The dashed line indicates the $95 \%$ confidence level upper limit on the number of observed events; no events were observed by either experiment. leading to the $95 \%$ confidence level upper limit at three events, shown as the dashed line. When intersected with the curves of expected events, the limits become $34 \mathrm{GeV}$ for OPAL, $32 \mathrm{GeV}$ for L3, $26 \mathrm{GeV}$ for DELPHI and $25 \mathrm{GeV}$ for ALEPH. One can even combine the limits of OPAL and L3 to raise this limit to $36 \mathrm{GeV}$.

In addition to this analysis, ALEPH [18] has recently used a new method to search for the Higgs. The missing energy and dilepton searches are limited towards higher masses by the fact that the Higgs decays become more spherical and their topology is not as clear anymore. A different approach which requires good knowledge of the detector response is to reconstruct the visible energy in the event with some precision. To this end ALEPH has developed the following algorithm: i) the charged energy as measured in the TPC was added together. ii) Isolated photons in the electromagnetic calorimeter were counted as neutral energy. iii) The remaining electromagnetic energy was corrected for the $e / \pi$ response ratio and added to the energy observed in the hadron calorimeter. If the so obtained hadronic energy exceeded the total charged energy, the excess was counted as neutral hadronic energy and added to the visible energy. This algorithm was checked using multihadronic events with a hard photon from initial state radiation. The visible hadronic energy in these events can be measured and also calculated from the photon energy. A comparison of the two gives the accuracy of the method which corresponds to a resolution of $6 \mathrm{GeV}$.

Figures $8 a$ and $b$ show the total visible energy spectrum expected for a $30 \mathrm{GeV}$ and $40 \mathrm{GeV}$ Higgs, respectively. Figure $8 \mathrm{c}$ shows the visible energy of the observed multihadron events. Note that the Monte Carlo spectra have an arbitrary scale. The number of expected events as a function of the Higgs mass is shown in figure 9. Using the observed upper limit of three events, ALEPH excludes the Higgs up to $40 \mathrm{GeV}$. In summary, the LEP experiments have excluded the mass range from zero to $40 \mathrm{GeV}$ for the production of Standard Model Higgs bosons. 

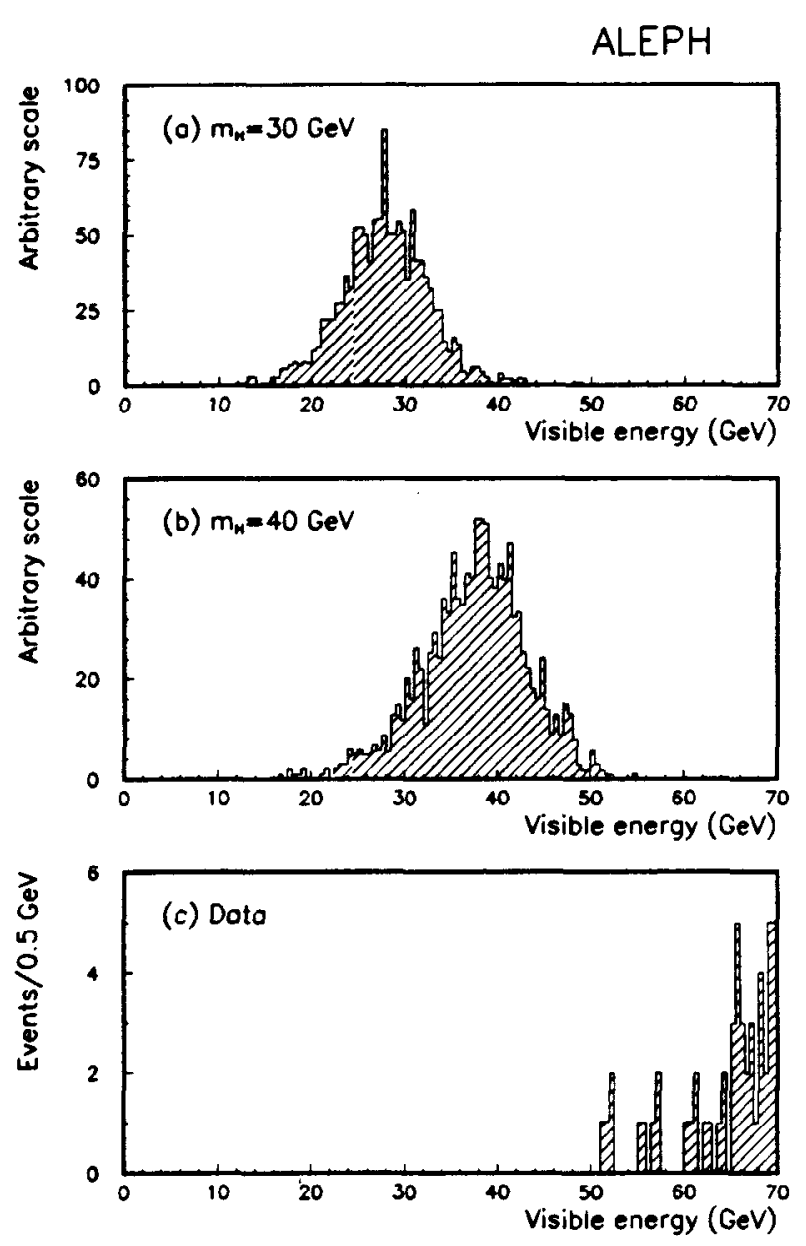

Figure 8: The spectrum of the reconstructed visible energy in the ALEPI detector. (a) A Monte Carlo simulation of a $30 \mathrm{GeV}$ Higgs, (b) a $40 \mathrm{GeV}$ Higgs, and (c) the observed events after some initial cuts. The distributions (a) and (b) are not normalised to the luminosity.

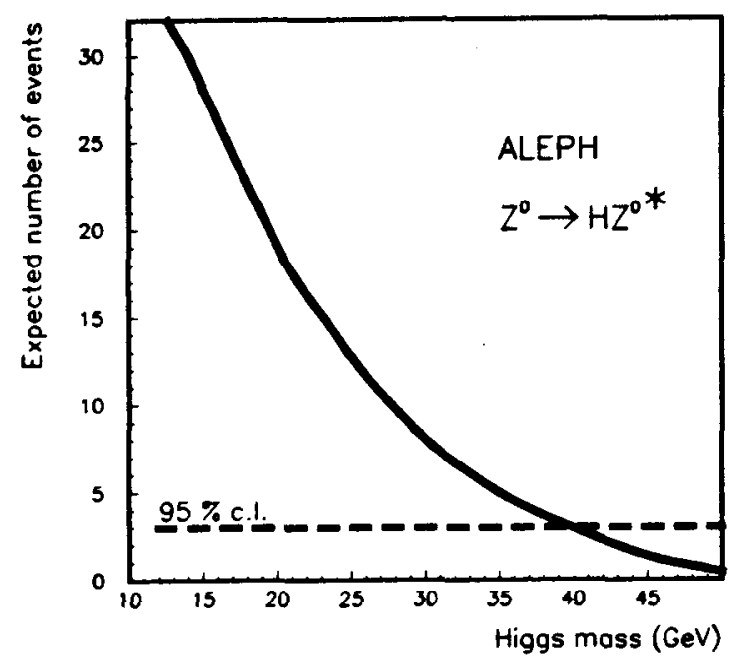

Figure 9: The number of events expected in the search based on the visible energy by ALEPH from the combined channels $\mathrm{HZ}^{\mathrm{O}^{*}} \rightarrow \mathrm{H} \nu \bar{\nu}$ and leading to the limit at $40 \mathrm{GeV}$. 


\subsection{Search for the Higgs in the Minimal Super Sym-} metric Extension to the Standard Model

In the Minimal Super Symmetric extension to the Standard Model (MSSM) not one but five physical Higgs states are predicted: a neutral scalar $\mathbf{h}^{0}$ with mass $m_{\mathrm{h}^{0}}$ below $m_{\mathrm{Z}}$, a neutral scalar $\mathrm{H}^{0}$ with $m_{\mathrm{H}^{0}}>m_{\mathrm{Z}}$, a neutral pseudoscalar $A^{0}$ with $m_{A^{0}}>m_{h^{e}}$ and two charged scalars $H^{ \pm}$with $m_{H^{*}}>m_{W}$. At current LEP energies we will therefore only be able to see the $h^{\circ}$ and $A^{0}$. In the MSSM there are four parameters of which only two are independent: $m_{h^{0}}, m_{A^{0}}, \beta$ and $\alpha$. Here $\alpha$ is the mixing angle of $h^{0}$ and $H^{0}$ and $\tan \beta=v_{2} / v_{1}$, where $v_{i}$ is the vacuum expectation value of the Higgs doublet $i$. Usually either $\left(m_{\mathrm{A}^{0}}, m_{\mathrm{h}^{0}}\right)$ or $\left(m_{\mathrm{h}^{0}}, \tan \beta\right)$ is chosen as free parameters.

The production rate of Higgs pairs is given by

$$
\frac{\Gamma\left(\mathrm{Z}^{0} \rightarrow \mathrm{h}^{0} \mathrm{~A}^{0}\right)}{\Gamma\left(\mathrm{Z}^{0} \rightarrow \nu_{\mathrm{e}} \bar{\nu}_{\mathrm{e}}\right)}=\frac{\lambda^{3}}{2} \cos ^{2}(\alpha-\beta)
$$

where $\lambda=\left[\left(1-x_{h}^{2}-x_{A}^{2}\right)^{2}-4 x_{h}^{2} x_{A}^{2}\right]^{1 / 2}$ is a kinematical factor with $x_{h}=$ $m_{\mathrm{h}^{\circ}} / m_{\mathrm{Z}}$ and $x_{A}=m_{\mathrm{A}^{0}} / m_{\mathrm{Z}}$. The factor $\cos ^{2}(\alpha-\beta)$ is purely related to the masses of the bosons:

$$
\cos ^{2}(\alpha-\beta)=\frac{m_{h^{0}}^{2}\left(m_{\mathrm{Z}}^{2}-m_{\mathrm{h}^{0}}^{2}\right)}{m_{\mathrm{A}^{0}}^{2}\left(m_{\mathrm{Z}^{2}}^{2}+m_{\mathrm{A}^{0}}^{2}-2 m_{\mathrm{h}^{0}}^{2}\right)} .
$$

The production rate of the single neutral scalar Higgs is given relative to the rate for production of the Standard Model Higgs:

$$
\frac{\Gamma\left(Z^{0} \rightarrow h^{0} Z^{0 *}\right)}{\Gamma\left(Z^{0} \rightarrow H_{S M} Z^{0 *}\right)}=\sin ^{2}(\beta-\alpha) \text {. }
$$

The limits on the latter can therefore be used to set limits on the MSSM Higgs.

The decay of the MSSM Higgs bosons depends on the value of $\tan \beta$ :

$$
\begin{array}{lcc}
\operatorname{Br}\left(h^{0} \rightarrow \tau^{+} \tau^{-}: c \bar{c}: b \bar{b}\right)=1: & 2.1 \cot ^{2} \alpha \cot ^{2} \beta & : 19 \lambda_{b}^{3} \\
\operatorname{Br}\left(A^{0} \rightarrow \tau^{+} \tau^{-}: c \bar{c}: b \bar{b}\right)=1: & 2.1 \cot ^{4} \beta & : 19 \lambda_{b}^{3},
\end{array}
$$

where $\lambda_{b}^{2}=1-4 m_{b}^{2} / m_{h^{0}}^{2} A^{0}$. For $\tan \beta>1$, the theoretically favoured case, the decay is mainly to $b \hat{b}$ with a $6 \%$ contribution of $\tau^{+} \tau^{-}$. For $\tan \beta<1$, the decay is mainly to $c \bar{c}$.

ALEPH [19], OPAL [16] and DELPHI [20] have each searched for the MSSM Higgs. The results can be combined to exclude the large area of the $\left(m_{\mathrm{b}}, \tan \beta\right)$ plane as shown in figure 10.[21] The high limit of $40 \mathrm{GeV}$ on the Standard Model Higgs by ALEPH excludes the area to the left of the solid line. The high mass region around $\tan \beta=1$ is excluded because $m_{A^{\circ}}$ is large for $\tan \beta \approx 1$, which is kinematically impossible.

The OPAL collaboration has analysed four-jet events, for the $Z^{0} \rightarrow$ $h^{0} \mathrm{~A}^{0} \rightarrow c \bar{c} c \bar{c}$ decay. Kinematical cuts were applied to the four-jet events to enhance a possible contribution from $h^{0} A^{0}$ decays. Ten events survived these cuts, 13.3 were predicted from a QCD Monte Carlo. No signal was found in these events, and OPA $\mathrm{L}$ was able to exclude the region indicated as '4-jet' in figure 10 .

The region indicated as $D^{*}$ at a somewhat lower mass was excluded by DELPHI, also using the $h^{0} A^{0} \rightarrow c \bar{c} c \bar{c}$ decay, but searching for the $D^{*}$ in four-jet events. The pion in the decay chain $D^{*} \rightarrow D^{0} \pi$ has a very low momentum transverse to the jet axis (typically $30 \mathrm{MeV}$ ). The $D^{*}$ therefore appears as an excess near zero in a plot of the transverse momentum of charged particles with respect to the jet axis. This $\mathrm{D}^{*}$ signal is present as expected in two-jet events, but not in four-jet events, leading to the excluded area in figure 10.

The upper corner of the plot is excluded by a search by DELPHI for events of the type $h^{0} A^{0} \rightarrow \tau^{+} \tau^{-} b \bar{b}$. A direct search for events with two jets and two leptons yielded no signal and excluded the region labeled $\tau^{+} \tau^{-} b \bar{b}$ in figure 10. The remaining area at low masses was excluded by a DELPHI analysis of two-jet events. In this mass range the Higgs bosons appear as collimated jets and can be distinguished from regular jets by the jet invariant mass and the fact that no string effects appear in the $h^{0} \mathrm{~A}^{0}$ decay of the $Z^{0}$.

When the limits from the three experiments are combined all values in the $\left(m_{h^{0}}, \tan \beta\right)$ plane can be excluded for $m_{h^{0}}<34 \mathrm{GeV}$. 


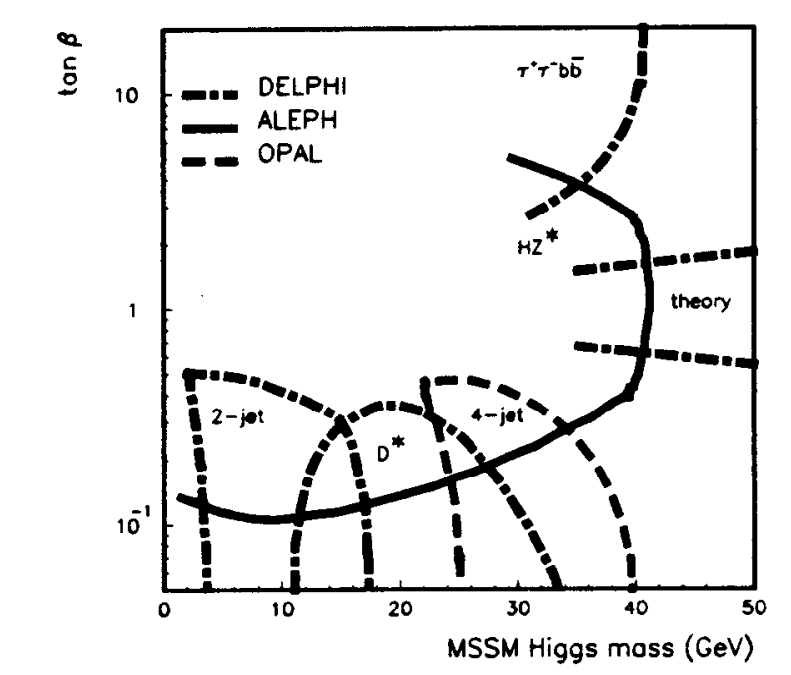

Figure 10: The parameter space of $\left(m_{\mathrm{h}^{0}}, \tan \beta\right)$ in the search for the MSSM Higgs. All areas except the upper and lower right corners of the plot are excluded by various searches described in the text.

\subsection{Excited Leptons and Quarks}

Searches for excited leptons and quarks were performed by OPAL [22] ALEPH [23] and L3. [24] The excited lepton is assumed to decay to a regular lepton under emission of a photon, and should therefore appear as a peak in the invariant mass spectrum of lepton- $\gamma$ combinations in lepton pair events. The searches for excited leptons $(l=e, \mu, \tau)$ were limited to two reactions:

which limits $m_{\mathrm{i}}$ - to $m_{\mathrm{Z}} / 2$, or

$$
e^{+} e^{-} \rightarrow l^{ \pm *} l^{\mp *} \rightarrow l^{ \pm} l^{\mp} \gamma \gamma
$$

$$
e^{+} e^{-} \rightarrow l^{ \pm *} l^{\mp} \rightarrow l^{ \pm} l^{\mp} \gamma,
$$

which allows $m_{i} \cdot$ up to $m_{\mathrm{z}}$. In addition, the process $e^{+} e^{-} \rightarrow e^{ \pm} e^{\mp *}$ can occur in the $t$-channel with little momentum transfer to one of the initial electrons, which therefore escapes detection. The event topologies searched for were either a lepton pair with one or two photons or a single electron with a photon. The excited leptons were assumed to be spin- $\frac{1}{2}$ particles with Standard Model $\left(\mathrm{Z}^{0}, \gamma\right)$ couplings. The backgrounds to these searches are the radiative lepton pairs and radiative Bhabha events. Table 2 summarises the results from the OPAL, ALEPH and $\mathrm{L} 3$ collaborations. In all cases the observed number of lepton-pair events with one photon agrees very well with the number expected from the Monte Carlo simulations. Also the numbers of $t$-channel $e^{+} e^{-} \gamma$ events are in good agreement. Note that the simulations predict no events with two photons at this level of statistics, whereas L3 observes three $e^{+} e^{-} \gamma \gamma$ events and OPAL and L3 each observe one $\mu^{+} \mu^{-} \gamma \gamma$ event. None of the invariant mass spectra show a significant signal, leading to the lower limits summarised in table 3 . These limits are at $95 \%$ confidence level and essentially rule out the existence of excited leptons up to $m_{\mathrm{Z}} / 2$.

In the reaction (11), a free parameter $f / \Lambda$ remains in the effective Langrangian, $f$ being the $\left(7^{0}, \gamma\right)-l^{ \pm *}$ coupling and $\Lambda$ the composite mass scale. The lower limits are therefore expressed in terms of $f / \Lambda$ as shown in figure 11 for the case of the excited muon. The results of ALEPH, OPAL and L3 are given together with earlier results from AMY and TOPAZ at the TRISTAN storage ring.[25] The results for $e^{*}$ and $r^{*}$ are similar. 


\begin{tabular}{|lr|ccc|cc|cc|}
\hline Exp. & \multicolumn{4}{|c|}{$e^{+} e^{-}$} & \multicolumn{2}{|c|}{$\mu^{+} \mu^{-}$} & \multicolumn{2}{|c|}{$\tau^{+} \tau^{-}$} \\
$\int L$ & & $\gamma$ & $\gamma \gamma$ & $t$-chan. & $\gamma$ & $\gamma \gamma$ & $\gamma$ & $\gamma \gamma$ \\
\hline OPAL & data & 29 & 0 & 2 & 19 & 1 & 27 & 0 \\
$1.15 \mathrm{pb}^{-1}$ & MC & 31 & 0 & 3 & 22 & 0 & 26 & 0 \\
\hline ALEPH & data & 6 & 0 & 10 & 2 & 0 & 1 & 0 \\
$0.4 \mathrm{pb}^{-1}$ & MC & 6.3 & 0 & 8.5 & 2.2 & 0 & 1.5 & 0 \\
\hline L3 & data & 15 & 3 & 4 & 21 & 1 & & \\
$1-1.5 \mathrm{pb}^{-1}$ & MC & 15 & 0 & 3.3 & 22 & 0 & \\
\hline
\end{tabular}

Table 2: Summary of lepton pair events with additional photons found by OPAL, ALEPH and L3. The integrated luminosities used in the search are given for each experiment.

\begin{tabular}{|l|ccc|}
\hline Exp. & $e^{*}$ & $\mu^{*}$ & $\tau^{*}$ \\
\hline OPAL & 44.9 & 44.9 & 44.9 \\
ALEPH & 44.6 & 44.6 & 41.2 \\
L3 & 45.0 & 45.3 & \\
\hline
\end{tabular}

Table 3: Summary of limits on the masses of excited electrons, muons and tau leptons from OPAL, ALEPH and L3. The limits are lower limits in $\mathrm{GeV}$ at $95 \%$ confidence level.

Figure 11: Limits on the production of excited muons. The excluded areas are to the right of the curves of the $\mu^{*}$ coupling as a function of its mass.

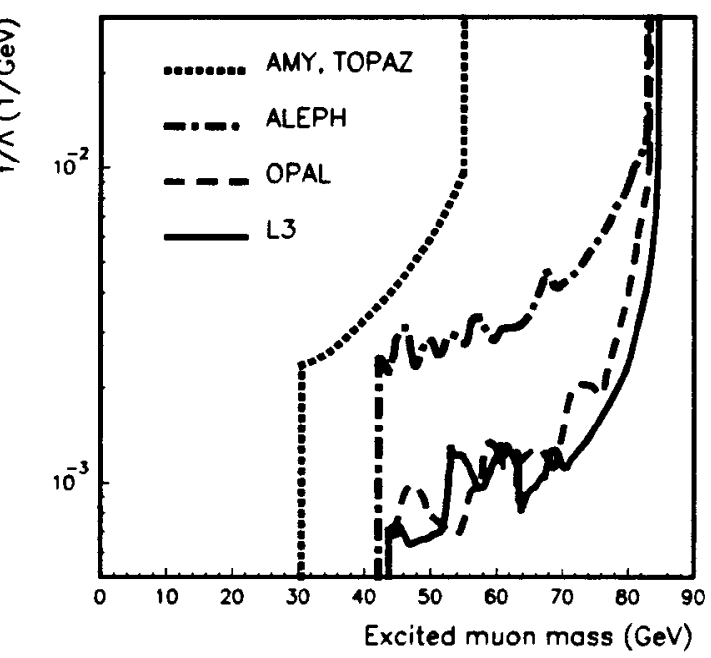


Searches for excited quarks were performed by OPAL [26] and ALEPH.[2] In these searches multihadronic events with an isolated photon were selected. OPAL required a photon energy greater than $10 \mathrm{GeV}$, isolated by more than $20^{\circ}$. In 77000 events, 78 candidates were found.

ALEPH required a photon energy greater than $10 \mathrm{GeV}$ with an isolation parameter as given in formula (4) larger than 2.5. Four such events were found in 11550 events, where two were expected.

These numbers are consistent with the expectation from initial and final state radiation. On this basis, OPAL gave an upper limit on the width of new radiative $Z^{0}$ decays of

$$
\Gamma\left(\mathrm{Z}^{0} \rightarrow \gamma+\mathrm{X}\right)<3.2 \mathrm{MeV} .
$$

In addition, upper limits on $b^{\prime}$ production were derived: OPAL found

$$
\frac{\Gamma\left(Z^{0} \rightarrow b^{\prime} b^{\prime}\right)}{\Gamma\left(Z^{0} \rightarrow \text { had }\right)} \times B r\left(b^{\prime} \rightarrow \gamma+X\right)<8.7 \times 10^{-4}
$$

and $A L E P H$ found that $m_{b^{\prime}}>46 \mathrm{GeV}$ under the assumption that $B R\left(b^{\prime} \rightarrow\right.$ $\gamma+X)>5 \%$.

\section{Conclusion}

No new particles were observed at LEP yet. The good agreement between the measured $Z^{0}$ parameters and their Standard Model values already places severe limits on the existence of new particles. However, direct searches are more sensitive and have led to limits close to the kinematical boundaries for new quarks, excited quarks and excited leplons.

Using a variety of search methods, all LEP collaborations have contributed to exclude the existence of the Standard Model Higgs boson in the mass range between zero and $40 \mathrm{GeV}$. In the context of the Minimal Super Symmetric extension to the Standard Model, the Higgs has been excluded up to $34 \mathrm{GeV}$ for all values of $\tan \beta$.
With an increase of the integrated luminosity and a more complete understanding of the detectors it will be possible to increase these limits substantially, even before the advent of LEP 200.

\section{References}

[1] E. Fernandez, talk presented at the Neutrino 90 conference at CERN, June 11, 1990.

[2] D. Decamp et al. (ALEPH Collaboration) Phys. Lett. B236, 511 (1990).

[3] G. Burgers and F. Jegerlehner, CERN 89-08, Vol. 1, p. 55 (1989)

[4] M. Z. Akrawy et al. (OPAL Collaboration), Phys. Lett. B236, 364 (1990).

[5] P. Abreu et al. (DELPHI Collaboration) Phys. Lett. B242, 536 (1990)

[6] J. F. Gunion, H. E. Haber, G. Kane and S. Dawson,

'The Higgs llunter's Guide', Addison-Wesley Publishing Company, Menlo Park, California (1990).

[7] F. A. Berends and R. Kleiss, Nucl. Phys. B260, 32 (1985).

[8] M.Consoli and W.Hollik, CERN 89-8, Vol. 1, p. 39 (1989)

[9] D. Decamp et al. (ALEPH Collaboration), Phys. Lett. B236, 233 (1990), and CERN-EP/90-70 (1990).

[10] M. Z. Akrawy et al. (OPAL Collaboration) CERN-PPE/90-116 (1990).

[11] P. Abreu et al. (DELPHI Collaboration), Phys. Lett. B342, 1 (1990).

[12] D. Decamp et al. (ALEPH Collaboration),

Phys. Lett. B241, 141 (1990) 
[13] M. Voloshin and V. Zakharov, Phys. Rev. lett. 45, 688 (1980) M. Voloshin, Sov. J. Phys. 44(3), 478 (1986).

S. Raby and G. B. West, Phys. Rev. D38, 3488 (1988)

[14] E. W. N. Glover and J. J. van der Bij, CERN 89-8, Vol. 2, p. 1 (1989).

[15] H. Kolanoski, 'Two-Photon Physics at $e^{+} e^{-}$Storage Rings', Springer-Verlag Berlin, (1984).

[16] M. Z. Akrawy et al. (OPAL Collaboration), CERN-EP/90-100 (1990), and OPAL internal note OPAL-PHYS-90/10B (1990).

[17] B. Adeva et al. (L3 Collaboration), L3 Preprint \# 10, June 23 (1990).

[18] D. Decamp et al. (ALEPH Collaboration), CERN-PPE/90-101 (1990).

[19] D. Decamp et al. (ALEPH Collaboration), Phys. Lett. B237, 291 (1990).

[20] P. A breu et al. (DELPHI Collaboration), Phys. Lett. 245B, 276 (1990), and DELPHI internal note, July 12 (1990).

[21] F.Richard, private communication.

[22] M. Z. Akrawy et al. (OPAL Collaboration), Phys. Lett. B242, 135 (1990).

[23] D. Decamp et al. (ALEPH Collaboration), Phys. Lett. B236, 501 (1990).

[24] B. Adeva et al. (L3 Collaboration), L3 Preprint \# 7, June 20 (1990).

[25] S. K. Kim et al. (AMY Collaboration), Phys. Lett. B223, 476 (1989). 1. Adachi et al. (TOPAZ Collaboration), Phys. Lett. B228, 553 (1989).

[26] M. Z. Akrawy et al. (OPAL Collaboration), Phys. Lett. B246, 285 (1990), and OPAL internal note OPAL-PHYS-007 (1990). 